I referee dell'annata 2009

\title{
Gianpaolo Abatecola
}

Ricercatore di Economia e gestione delle imprese nell'Università "Tor Vergata" di Roma

\section{Guido Alpa}

Professore ordinario di Diritto civile nell'Università "La Sapienza” di Roma

\section{Nicoletta Buratti}

Professore associato di Marketing nell'Università di Genova

\section{Roberto Cafferata}

Professore ordinario di Economia e gestione delle imprese nell'Università "Tor Vergata" di Roma

\section{Francesco Capriglione}

Professore ordinario di Diritto degli intermediari e dei mercati finanziari nell'Università "Luiss G. Carli" di Roma

\section{Clara Caselli}

Professore ordinario di Economia e gestione delle imprese nell'Università di Genova

\section{Marcella De Martino}

Ricercatore presso l'Istituto di ricerche sulle attività terziarie del Cnr di Napoli

\section{Claudio Ferrari}

Professore associato di Economia dei trasporti nell'Università di Genova

\section{Mario Grasso}

Docente di Economia e gestione delle imprese turistiche nell'Università di Genova

\section{Renato Midoro}

Professore ordinario di Economia e gestione delle imprese marittime e portuali nell'Università di Genova

\section{Alfonso Morvillo}

Direttore dell' Istituto di ricerche sulle attività terziarie del Cnr di Napoli

\section{Paola Paniccia}

Professore ordinario di Economia e gestione delle imprese nell'Università "Tor Vergata" di Roma

\section{Paolo Parini}

Professore ordinario di Economia e gestione delle imprese di servizi nell'Università di Genova 


\section{Clara Petrillo}

Ricercatore presso l'Istituto di ricerche sulle attività terziarie del Cnr di Napoli

\section{Sara Poggesi}

Dottore di Ricerca in Economia e gestione delle aziende e delle amministrazioni pubbliche nell'Università “Tor Vergata” di Roma

\section{Francesco Scafarto}

Ricercatore in Economia e gestione delle imprese nell'Università “Tor Vergata" di Roma

\section{Goffredo Sdrubolini}

Collaboratore del Dipartimento di economia e metodi quantitativi nell'Università di Genova

\section{Barbara Sibilio Parri}

Professore ordinario di Economia aziendale nell’Università di Firenze

\section{Mariapina Trunfio}

Ricercatore in Economia e gestione delle imprese turistiche nell'Università "Parthenope" di Napoli

\section{Marco Valeri}

Dottorando di ricerca in Economia e organizzazione delle imprese nell'Università "Tor Vergata" di Roma

\section{Mario Venturino}

Docente in Programmazione e controllo degli intermediari finanziari nell'Università di Genova

\section{Giuseppe Vito}

Docente di Economia e gestione delle imprese marittime nell'Università "Parthenope" di Napoli 